\title{
INFRECUENTE PRESENTACIÓN CLINICOPATOLÓGICA DEL DAÑO TUBULAR AGUDO POR CADENAS LIVIANAS EN EL MIELOMA MULTIPLE
}

\section{INFREQUENT CLINICOPATHOLOGICAL PRESENTATION OF ACUTE TUBULAR DAMAGE BY LIGHT CHAINS IN MULTIPLE MYELOMA}

\author{
Erika E. Faure ${ }^{1}$, Julieta Imahorn ${ }^{1}$, Natalia Saavedra ${ }^{1}$, Ana María Sesín ${ }^{2}$, María Elisa Dionisio de Cabalier ${ }^{1}$, \\ Jorge H. Mukdsi, ${ }^{1,3}$.
}

\section{Resumen:}

El mieloma múltiple (MM) es la segunda neoplasia hematológica más común. Reportamos un caso de tubulopatía por cristales en un contexto de proteinuria de rango nefrótico. Este es el segundo caso reportado de tubulopatía en el mieloma múltiple sin síndrome de Fanconi.

El mieloma múltiple (MM) es la segunda neoplasia hematológica más común que puede comprometer los riñones, siendo el principal factor fisiopatológico de tal afección la secreción de paraproteínas monoclonales, que pueden ser glomerulopáticas o tubulopáticas ${ }^{1-3}$. Reportamos un caso de tubulopatía por cristales en un contexto de proteinuria de rango nefrótico ( $>6 \mathrm{~g} / 24 \mathrm{~h})$ e insuficiencia renal $(4 \mathrm{mg} / \mathrm{dL})$ en una paciente de 68 años de edad. A nivel de la biopsia renal se apreciaron 18 glomérulos histológicamente normales. Los túbulos proximales presentaron cambios de daño epitelial con lúmenes distendidos y epitelio aplanado con vacuolización, e inclusiones intracelulares pálidas con hematoxilina/eosina (Figura 1A-B). El componente vascular presentó leve arterioloesclerosis hialina. La tinción de Rojo Congo fue negativa. La muestra para inmunofluorescencia solo incluyó músculo esquelético. A nivel ultraestructural se observaron a nivel del epitelio tubular inclusiones cristalinas con apariencia fibrilar dispuestas en grupos paralelos (Figura 1C-D). Los glomérulos no exhibieron cambios subcelulares de significación. Con posterioridad la paciente se diagnostica de padecer una proliferación neoplásica de células plasmáticas. Este es el segundo caso reportado de tubulopatía en el mieloma múltiple sin síndrome de Fanconi ${ }^{1}$. Lahlein fue el primero en reportar una tubulopatía proximal por inclusiones en pacientes con $\mathrm{MM}^{4}$.

Sin embargo, la proteinuria nefrótica no se observa cuando la lesión compromete el epitelio tubular proximal como se evidencia en nuestro caso ${ }^{1}$.

Según Larsen y col$^{5}$, es muy difícil determinar el papel de los túbulos proximales en la proteinuria de nuestra paciente. Sin embargo, se ha sugerido que la saturación del transporte mediado por cubilina y megalina podría ser uno de los mecanismos causales de la proteinuria.

1 Cátedra de Anatomía Patológica. Facultad de Ciencias Médicas. Universidad Nacional de Córdoba. Córdoba. Argentina

2 Servicio de Nefrología-Hospital Nacional de Clínicas-Facultad de Ciencias Médicas. Universidad Nacional de Córdoba. Córdoba. Argentina

3 Email de contacto: mukdsijorge@gmail.com 


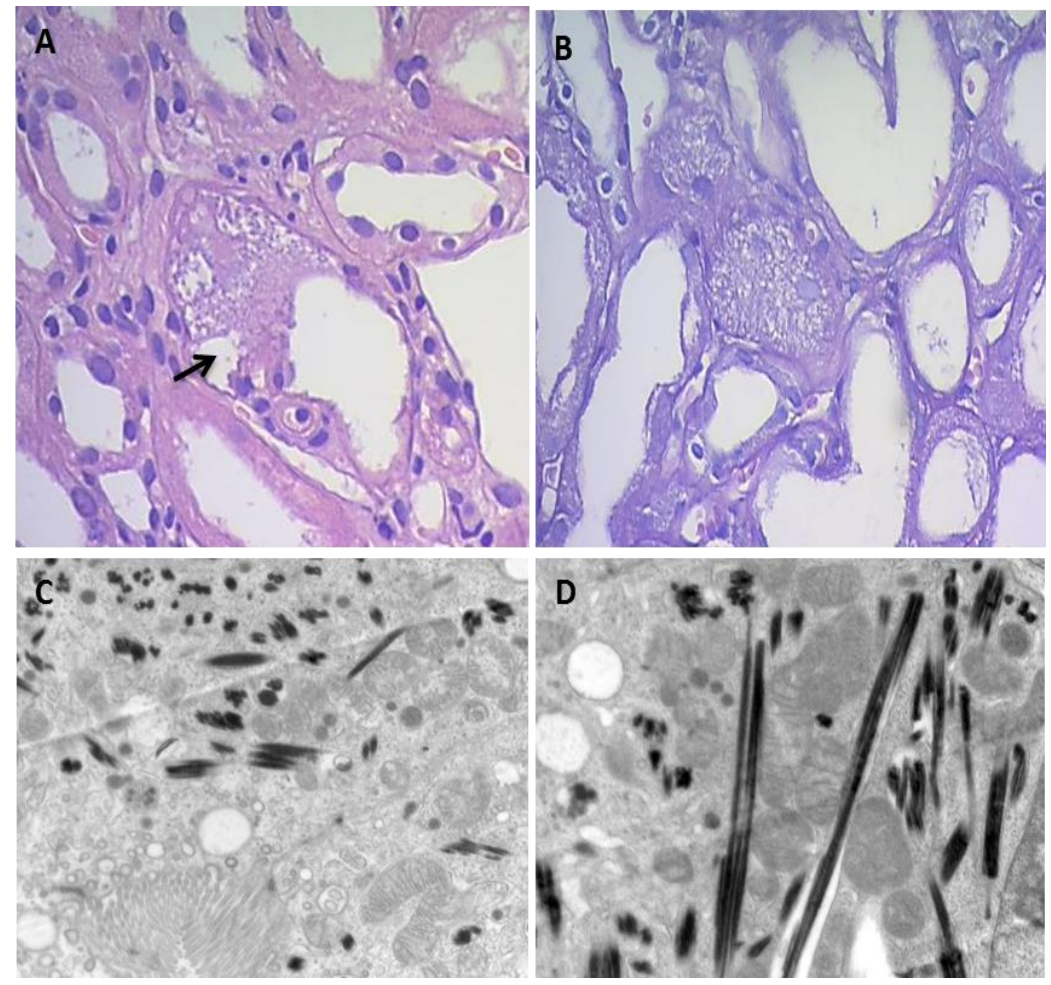

Figura 1 A y B. Daño tubular agudo y vacuolización (flecha) del epitelio tubular (A: Hematoxilina/Eosina) y negativo en ácido periódico de Schiff (B). 500X. C y D: Microfotografías electrónicas de transmisión que muestran túbulos proximales (C-3000X) con grandes cristales en forma de varilla (D-6000X).

\section{Bibliografía}

1. Stompór T, Perkowska-Ptasińska A, Wojciechowska M, et al. Unusual manifestation of crystalline light chain tubulopathy in patient with multiple myeloma: case report and review of the literature. Ren Fail 2014;36:795-9

2. Nasr SH, Valeri AM, Sethi S, et al. Clinico-pathologic correlations in multiple myeloma: a case series of 190 patients with kidney biopsies. Am J Kidney Dis 2012;59:786-94

3. Korbet SM, Schwartz MM. Multiple myeloma. J Am Soc Nephrol 2006;17:2533-45

4. Lahlein M. Euweisskrystalle in den harnkanalchen bei multiplen myelom. beitr zi Path Anat Vz Allg Path 1921;69:295

5. Larsen CP, Bell JM, Harris AA, et al. The morphologic spectrum and clinical significance of light chain proximal tubulopathy with and without crystal formation. Mod Pathol 2011;24:146-54 